\title{
Method for Fabricating Miniaturized NiTi Self-Expandable Thin Film Devices with Increased Radiopacity
}

\author{
Christoph Bechtold $^{1}$ Rodrigo Lima de Miranda ${ }^{1}$ Christoph Chluba ${ }^{1,2}$. \\ Christiane Zamponi $^{1,2} \cdot$ Eckhard Quandt ${ }^{2}$
}

Published online: 21 November 2016

(C) ASM International 2016

\begin{abstract}
Nitinol is the material of choice for many medical applications, in particular for minimally invasive implants due to its superelasticity and biocompatibility. However, NiTi has limited radiopacity which complicates positioning in the body. A common strategy to increase the radiopacity of NiTi devices is the addition of radiopaque markers by micro-riveting or micro-welding. The recent trend of miniaturizing medical devices, however, reduces their radiopacity further, and makes the addition of radiopaque markers to these miniaturized devices difficult. NiTi thin film technology has great potential to overcome such limitations and to fabricate new generations of miniaturized, self-expandable NiTi medical devices with additional functionalities, such as structured multilayer devices with increased radiopacity. For this purpose, we have produced superelastic thin film NiTi samples covered locally with Tantalum structures of different thickness and different shape. These multilayer devices were characterized regarding their mechanical and corrosion properties as well as their X-ray visibility. The superelastic behavior of the underlying NiTi layer is impeded by the Ta layer, and shows therefore a dependence on the Tantalum patterning geometry and thickness. No delamination was observed after mechanical and corrosion tests. The multilayers reveal excellent corrosion resistance, as well as a significant increase in radiopacity.
\end{abstract}

Christoph Bechtold

bechtold@acquandas.com

Acquandas GmbH, Kaiserstrasse 2, 24143 Kiel, Germany

2 Institute for Materials Science, Faculty of Engineering, University of Kiel, Kaiserstrasse 2, 24143 Kiel, Germany
Keywords $\mathrm{NiTi}<$ materials $\cdot$ Shape memory films Biocompatibility $\cdot$ Shape memory stents $\cdot$ Pseudoelasticity Radiopacity $\cdot$ Corrosion resistance

\section{Introduction}

Undoubtedly, Nitinol is a material with tremendous properties: its excellent mechanical and corrosion properties and its biocompatibility make it suitable, in particular, for use as material for minimally invasive medical devices since it enables crimping into small catheter sizes, easy deployment even through vessels with small bending radii, and good wall apposition [1]. Despite these tremendous properties, one major drawback of Nitinol is its poor radiopacity. Approaches to increase radiopacity have been manifold in the last two decades: the optimization of X-ray imaging parameters for scanning Nitinol devices has been investigated to begin with, in particular combinations of pulse-dose and frequency for achieving improved image quality. However, no significant improvements of objective or subjective stent detection rate or visibility were obtained [2]. Hence, the material itself requires adjustments. Different ternary alloys of NiTi-X have been investigated, the most promising ones being NiTiPt, NiTiPd [3-7], and NiTiW [8, 9]. All of these alloys show increased radiopacity and good corrosion properties; however, aside from a potential shift in transformation temperature, these alloys have either expensive additives or reduce the recoverable strain to around $30 \%$ of that of binary Nitinol. Other approaches involve composite materials, e.g., $\mathrm{NiTi}+\mathrm{Pt}$ drawn filled tubes with Pt content up to $30 \%$ [10]. These tubes substantially increase $\mathrm{X}$-ray visibility while maintaining good mechanical properties. Tantalum is another element that is often used for increasing 
radiopacity. Alloys consisting of NiTiTa, however, exhibit an increased transformation temperature, and are rather used for actuator applications $[11,12]$. Hence, Tantalum is often integrated in elemental form to the Nitinol structure, e.g., reservoirs can be created on the stent struts by laser ablation, filled with Ta powder, and closed by laser weldings [13, 14]. Most often, however, Nitinol stents are either equipped with eyelid structures and markers of $\mathrm{Au}, \mathrm{Ta}$, or Pt are micro-riveted into that eyelid structure, as implemented in the SMART ${ }^{\mathrm{TM}}$ stent by Cordis, or with pads to which the Tantalum markers are welded to [15-17]. X-ray investigations do clearly find that stent radiopacity is increased significantly with the help of radiopaque markers [18]. Ta markers are beneficial also in terms of corrosion resistance, since Ta is close to NiTi in the galvanic series. In contrast, markers of more noble materials, such as Au or $\mathrm{Pt}$, presumably have to be coated with a thin polymer layer [19] in order to protect the less noble NiTi from corrosion.

The corrosion of binary NiTi itself has been the subject of many studies and is an important aspect for determining the biological safety of the material [19-22]. Surface treatment and quality are in this respect pivotal parameters, and smooth surfaces with a homogeneous, defect-free corrosion-resistant titanium oxide surface layer reveal low corrosion rates and low Ni release, as well as high breakdown potentials [23, 24], and are consequently well suited for use as implant material surface. Shot-peened or sandblasted surfaces are interesting, since their higher roughness can be exploited to alter cell proliferation [25], but on the other hand, thus treated wires reveal lower corrosion resistance, presumably due to surface defects and an inability to remove the original scaling of the black oxide, which is a result of heat treatments during wire processing, by that method.

Furthermore, during NiTi ingot melting, carbide and oxide inclusions are introduced into the material, which also affect the corrosion behavior of the alloy, since a fraction of these inclusions are present at the surface. Vacuum arc remelting (VAR) and vacuum induction melting, followed by VAR (VIM/VAR), are two important melting methods. Analysis of medical-grade tubes fabricated by these methods reveals that a larger inclusion size and quantity correlates with a higher fraction of breakdowns in potentiodynamic polarization test [26]. It was shown that voids that occur at inclusion particles during thermomechanical processing can act as micro-crevices in contact with electrolyte and thus promote localized corrosion. The degree of cold or hot work during tube drawing alters size and distribution of inclusions, and therefore also affects breakdowns during corrosion tests. During each tube drawing step, inclusions are further fractionalized and the inclusion size decreases. Since the fraction of breakdowns correlates rather with inclusion size than with inclusion quantity, the resistance against localized corrosion improves with increasing number of tube drawing step [27]. Sputtered NiTi thin films reveal, in this respect, exciting results due to their complete lack of carbide or oxide inclusions. Consequently, no electrical breakdown was observed for sputtered films up to $1 \mathrm{~V}$ [26]. It is presumed that sputtered films have, in addition to the absence of inclusions, a homogeneous native oxide layer of a few tens of nm thickness that further increases corrosion resistance, despite a lack of surface treatment during processing.

More care must be taken regarding corrosion behavior when combinations of NiTi with a radiodense material are used, in particular, when more noble metals are involved which can trigger pitting corrosion at low breakdown potentials [28]. However, it was shown that NiTi with welded Ta markers, electropolished with optimized electrolyte solutions for this specific material combination, has smooth surfaces and reveals high breakdown potential comparable to that of pure NiTi [29].

In this study, we pursued an approach based on a MEMS fabrication process. This process allows for producing highly pure superelastic NiTi thin films up to $80 \mu \mathrm{m}$ thickness by magnetron sputtering, UV lithography, and wet chemical etching, and is explained in detail in [30]. The versatility of this MEMS fabrication process also allows for complex 2.5 D NiTi geometries, e.g., for use in various medical applications such as reservoirs for drugeluting systems, interface and tissue engineering, or micropumps [31]. Furthermore, the usage of different materials (insulators, conductive or magnetic materials, antibacterial or bioresorbable materials, materials for temperature probes) in combination with NiTi opens up new possibilities for creating additional device features and functionalities to a self-expandable device. Examples are intravascular stents with electrodes for sensing, stimulating, or ablating tissue; medical devices with local heating elements; antennas; temperature probes; drug-eluting mechanisms, etc. The issue of poor NiTi radiopacity can be addressed by adding Ta structures to the NiTi thin film, with the freedom of patterning Ta with high resolution where required and optimized Ta thickness, see Fig. 1. For a systematic investigation of the influence of Ta layers on the mechanical properties, X-ray visibility, and corrosion properties, which is subject of this study, we fabricated a series of test specimen covered with different Ta geometries and thickness (see Table 1; inset of Fig. 2).

\section{Methods and Fabrication}

The basis of the processing sequence is explained in detail in [30]. We pre-structured the sacrificial layers on nonoxidized 4" silicon wafers lithographically by spin coating a positive photoresist, followed by UV exposure on a Karl 

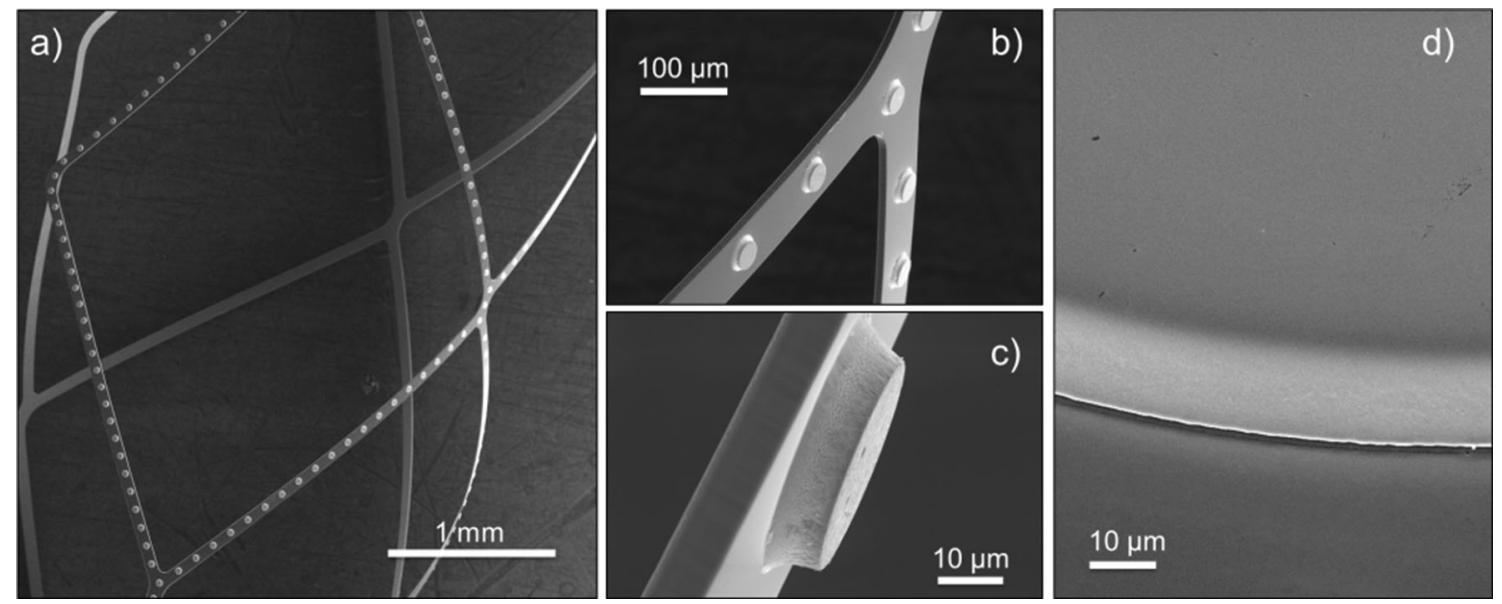

Fig. 1 a-c Stent-like structure covered with Ta dots after heat treatment in different magnifications; bright appearance of dots in SEM images due to increased Z-contrast. d Ta dot with rounded edges after surface finishing

Suss mask aligner MJB4 in hard contact mode, development, and wet chemical etching. NiTi is deposited from a 4" NiTi alloy target with an Alcatel 450 magnetron sputtering system up to a thickness of $35 \mu \mathrm{m}(3.9 \mathrm{~nm} / \mathrm{s}$ sputtering rate). The Ta layer is also sputter-deposited (between 10 and $20 \mu \mathrm{m}$ thickness) and structured using a second lithography step. Subsequently, the device is annealed in a high-vacuum chamber in order to avoid oxidation during the annealing process. During heat treatment, the amorphous NiTi structure is crystallized to obtain superelasticity. Heat treatment was carried out by means of a halogen lamp-driven rapid thermal annealing system (Createc Fischer RTA-6 SY09). Mechanical characterization was performed in a ZwickRoell Z0.5 testing machine with $100 \mathrm{~N}$ force gauge and temperature chamber. A self-built micro tensile tester consisting of an actuator (Pi M226-26S) and a Dysinet load cell DS011A9-100 N was mounted under a Zeiss Axiomat polarization microscope which enabled the simultaneous acquisition of stress-strain curves and surface images of the tensile specimen. A Gamry interface 1000 potentiostat was used for corrosion tests. The latter was conducted in Hanks' solution using a three-electrode setup with a platinum sheet $(7 \mathrm{~mm} \times 40 \mathrm{~mm})$ as counter electrode and a $\mathrm{Ag} / \mathrm{AgCl}$ reference electrode (Haber-Luggin capillary), in accordance to ASTM standard [32]. A constant temperature of $37{ }^{\circ} \mathrm{C}$ and a constant $\mathrm{pH}$ value of 7.4 were maintained throughout the experiment, the latter was controlled using a carbon dioxide-sodium bicarbonate $(0.35 \mathrm{~g} / \mathrm{l})$ buffer system. A more detailed description of the corrosion setup can be found in [33]. XRD investigations of sputtered Ta films were performed with a Seifert XRD 3003 4-circle system with $\mathrm{Cu} \mathrm{K}_{\alpha}$ radiation. The X-ray visibility tests were performed with a Siemens Fluorospot compact.

\section{Results and Discussion}

For X-ray visibility tests, test structures as shown in the inset of Fig. 2 were fabricated, with three rows of Ta dots with increasing diameter (from left to right), followed by three stripes. Three different Ta thicknesses $(10,15$, and $20 \mu \mathrm{m})$ were deposited. The Ta pattern is visible in all three radiographs; however, they become more distinct

Table 1 Overview of $20-\mu \mathrm{m}$-thick Ta structures patterned on NiTi

\begin{tabular}{|c|c|c|c|c|c|c|}
\hline \multirow[t]{2}{*}{ Ta geometry } & \multicolumn{3}{|c|}{ Dogbones $(0.5 \mathrm{~mm} \times 11 \mathrm{~mm})$} & \multicolumn{3}{|l|}{ Corrosion (10 mm diameter) } \\
\hline & $\varnothing / \mu \mathrm{m}$ & $\#$ & $\begin{array}{l}\text { Ta surface } \\
/ \mathrm{mm}^{2}\end{array}$ & $\varnothing / \mu \mathrm{m}$ & $\#$ & $\begin{array}{l}\text { Ta surface } \\
/ \mathrm{mm}^{2}\end{array}$ \\
\hline 1 Dots & 25 & 480 & 0.2356 & 25 & 23906 & 11.73 \\
\hline 2 Dots & 100 & 51 & 0.4006 & & & \\
\hline 3 Dots & 200 & 36 & 1.1310 & & & \\
\hline 4 Dots & 280 & 30 & 1.8473 & 280 & 193 & 11.88 \\
\hline 5 Stripe & $0.252 \mathrm{~mm} \times 13.00 \mathrm{~mm}$ & 1 & 3.2663 & $0.252 \mathrm{~mm} \times 4.729$ to $9.000 \mathrm{~mm}$ & 7 & 12.68 \\
\hline
\end{tabular}


with increasing Ta thickness. For $20-\mu \mathrm{m}$ Ta, the pattern can be clearly recognized, also by the difference in gray value. For image analysis, squares of $300 \times 300$ pixels have been extracted, and the difference between the mean gray value of the image and a rectangular area, ranging from left to right, containing the NiTi-Ta test pad, was calculated. Brightness and contrast of the original X-ray image were not changed. As expected, the difference in gray value increases with increasing Ta thickness and reaches $\sim 15 \%$ for $20-\mu \mathrm{m}$ Ta structures. Figure $2 \mathrm{c}$ shows the same behavior for $10-\mu \mathrm{m}$-thick NiTi stent structures. Without $\mathrm{Ta}$, this stent structure is invisible in the radiograph. Ta in rectangular shapes $(20 \mu \mathrm{m} \times 300 \mu \mathrm{m}, \sim 600$ rectangles $)$ was deposited on certain areas of the stent struts, again with different Ta thicknesses of 10,15 , and $20 \mu \mathrm{m}$. The radiographs show the increasing radiopacity of the multilayer stents.

Elementary Tantalum exhibits two crystalline phases which differ significantly in their mechanical properties. However, the alpha phase (body center cubic) is ductile, and the beta phase (tetragonal) is brittle. Consequently, for the use as radiodense material in combination with a selfexpandable medical device, the alpha phase is preferred. The sputtered Tantalum of this study was investigated by $\mathrm{X}$-ray diffraction (not shown) and revealed the alpha phase. No sign of the beta phase was observed; however, a mixture of both phases is often present in sputtered films [34]. For mechanical tests, sputtered dogbone structures with different Ta geometries were patterned, with a NiTi thin film thickness of $35 \mu \mathrm{m}$ and a Ta thickness of $20 \mu \mathrm{m}$. An overview over the dimensions of the Ta geometry is given in Table 1. The dot size varied from $25 \mu \mathrm{m}$ diameter to 100, 200, and $280 \mu \mathrm{m}$, see Fig. 3. Polarization microscopy during tensile loading reveals the austenite-martensite interface, and thus gives powerful insights into the transformation characteristics. For the $25-\mu \mathrm{m}$ Ta dots, a single interface was observed. This interface moved homogeneously from right to left for increasing strains, without any indications of an influence of the Ta dots on the interface movement. The angle of the interface was measured to be $59^{\circ}$ to strain direction, which is a typical angle found for Lüder-like shear deformation bands in polycrystalline NiTi specimen $[35,36]$. During strain release, the interface moves homogeneously in reverse direction. Signs of remanent martensite with increased surface roughness were not observed after a few cycles, though the small size of the dots would be expected to cause only small surface areas of increased roughness, which are difficult to detect with the given magnification. The transformation characteristics change significantly for Ta dots of $100 \mu \mathrm{m}$ thickness. As can be observed from the polarization microscopy images, multiple austenite-martensite interfaces appear, originating either directly before or after a $\mathrm{Ta}$ dot, in a $55^{\circ}$ angle. More interfaces are created when strain is increased, and simultaneously the area of existing martensite variants grows further, encompassing a larger fraction of the surrounding Ta dot. When further increasing the strain, the underlying NiTi thin film seems to be fully transformed into martensite, however, with remaining stress fields around the Ta dots with increased surface roughness. On releasing the strain, the NiTi structure with $100-\mu \mathrm{m}$ Ta dots returns fully to the austenite state. The influence of the $200-$ and $280-\mu \mathrm{m}$


(c)
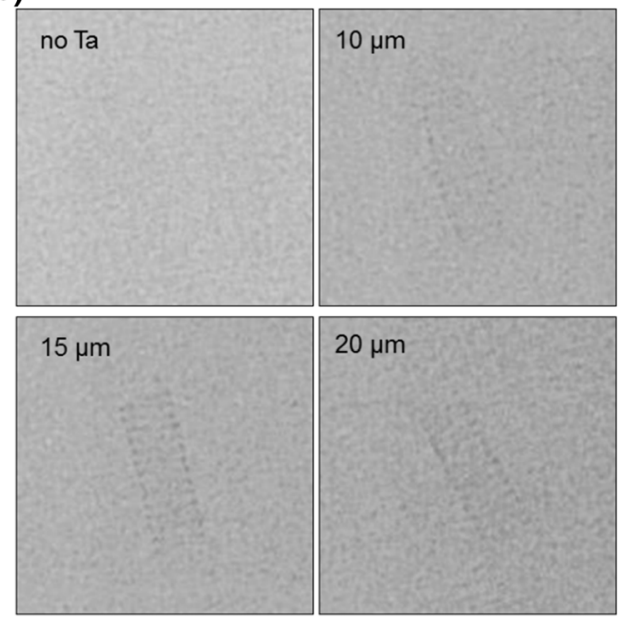

Fig. 2 a X-ray images of NiTi pads (5 $\mathrm{mm}$ in length) covered with different Ta geometries (see inset). Ta thickness was varied from 10 to 15 to $20 \mu \mathrm{m}$. b Analysis of the gray values of the X-ray images reveals an increased contrast for increasing Ta thickness. For $20 \mu \mathrm{m}$
Ta thickness, the structures are clearly visible in the X-ray images. c $10-\mu \mathrm{m}$ NiTi stent structure covered locally with different Ta thickness, Ta was deposited in a rectangular shape $(20 \mu \mathrm{m} \times 300 \mu \mathrm{m})$ on a selection of stent struts (Color figure online) 

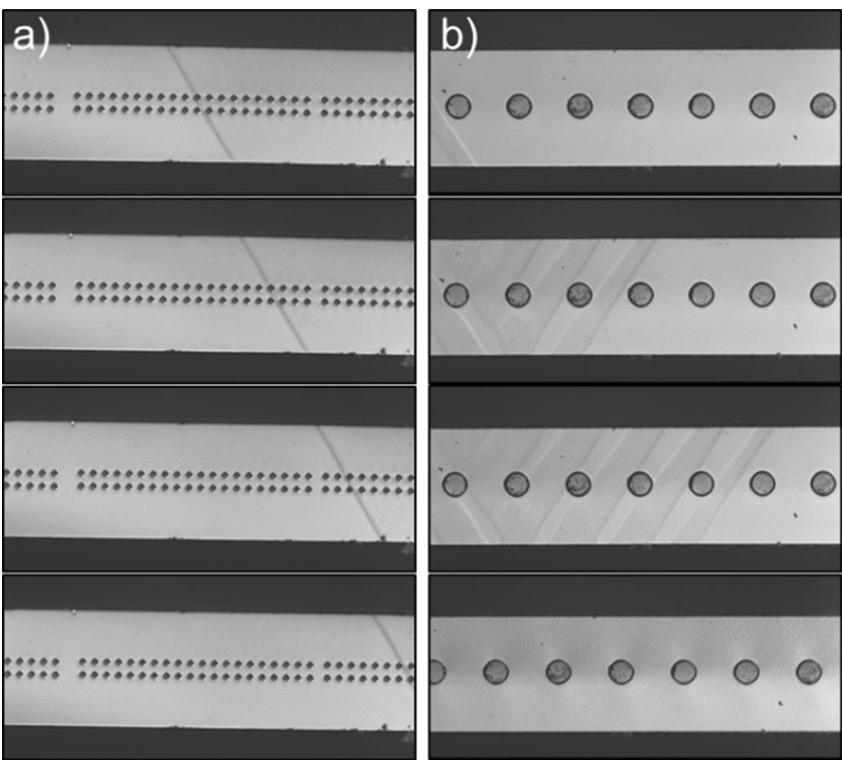

Fig. 3 a Two rows of $25-\mu \mathrm{m}$ Ta dots; a single austenite-martensite interface moves at $59^{\circ}$ angle from right to left during forward and from left to right during reverse transformation. b $100-\mu \mathrm{m}$ Ta dots; multiple martensite variants start to form in between Ta dots, new

Ta dots is more pronounced. Again, austenite-martensite interfaces are created in between the Ta dots, but the fraction of NiTi that is free to transform is significantly reduced. Also, no complete transformation into the martensite was observed, with areas of remaining austenite present at the projections of dots at $55^{\circ}$ angle. $5 \%$ strain could not be reached for these specimens due to film breakage originating at the edge of the dots. After repeating several cycles to $3 \%$ strain, regions with increased surface roughness were observed close to the dots along the main axis, presumable due to the complex stress fields generated by the Ta dots during straining and transformation into the martensitic phase. Clearly, amount, geometry, and thickness of the Ta layer play a pivotal role in influencing the superelastic transformation of the underlying $\mathrm{NiTi}$ structure. A dot geometry is disadvantageous in this respect, since it causes inhomogeneous stress fields, and the volume fraction of material that is free to transform is highly reduced. FEM calculations are a feasible tool for the optimization of Ta geometries, taking into account the angle towards strain direction at which the austenitemartensite interfaces appear. It must be noted that the total surface area covered with Ta is obviously different for the fabricated samples and increases for the samples with $25-\mu \mathrm{m}$ Ta dots (minimum amount of Ta) to samples with a continuous Ta stripe (maximum amount of $\mathrm{Ta}$ ), see Table 1. This fact must also be taken into account when assessing the influence of the fabricated Ta structures, and it is therefore the combination of reduced Ta diameter size and total surface covered with Ta that allows the austenite-
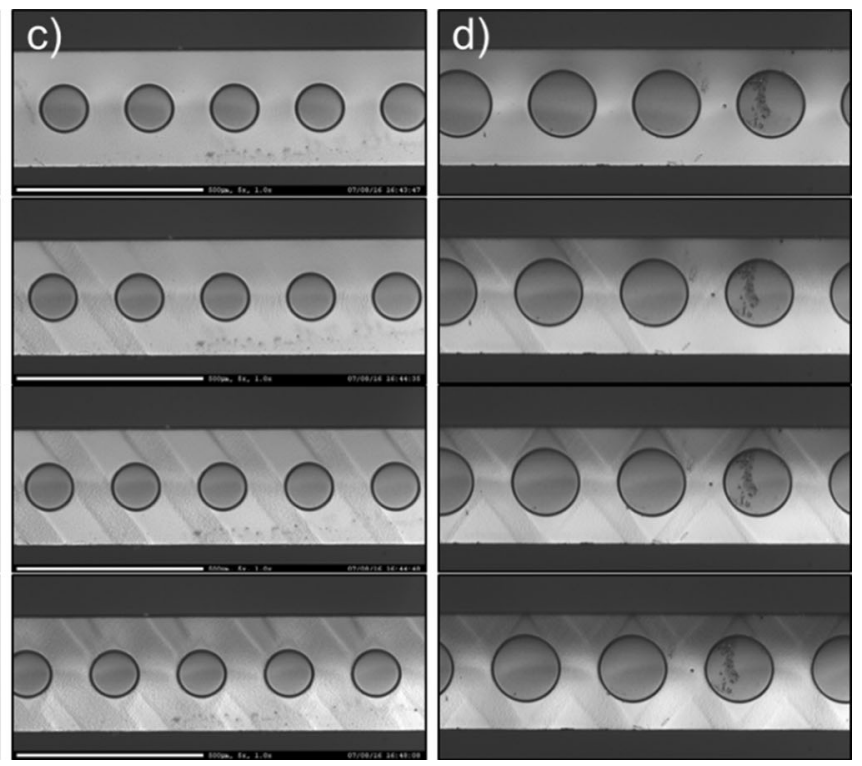

interfaces are created at $55^{\circ}$ and $-55^{\circ}$ angle, specimen still transforms completely into martensite. c $200-\mu \mathrm{m}$ Ta dots. d $280-\mu \mathrm{m}$ Ta dots; material does not transform completely into the martensite phase

martensite interface to move freely in the $25-\mu \mathrm{m}$ Ta dot samples.

Consequently, tensile curves for the fabricated samples differ, Fig. 4. For Ta dots with $25 \mu \mathrm{m}$ diameter, a smooth upper plateau up to $5 \%$ strain, and a smooth lower plateau were observed. Tensile curves are similar to pure NiTi thin film curves, see Fig. 5c). On the other hand, the upper plateau of samples with 200- and $280-\mu \mathrm{m}$ Ta dots becomes significantly more rugged and sloped. The ruggedness of the plateau is a result of the energy that is required to create new interfaces in between the Ta dots during straining. However, a smooth lower plateau remains for the samples with larger Ta dots during tensile tests up to $3 \%$ strain, since no new interfaces have to be created on releasing the strain, but interfaces already exist. It seems that the plateau slope is also influenced by the increasing size of the Ta dots. However, the fabricated samples are subject to a thickness variation of $\pm 14 \%$, which is a systematic thickness inhomogeneity of the prototyping (low volume) sputtering system used for this study, and equally influences plateau slope for pure NiTi thin films. The NiTi specimen covered with a single Ta stripe of $250 \mu \mathrm{m}$ width (50\% of dogbone width) did not show a transformation at all.

SEM investigations of the tensile specimen after ten tensile cycles revealed good adhesion of the Ta layer to the NiTi thin film. No delamination was observed in any of the samples, which includes samples that were loaded until film breakage. Breakages were observed exclusively in close vicinity of the Ta dots, with a tendency of following 


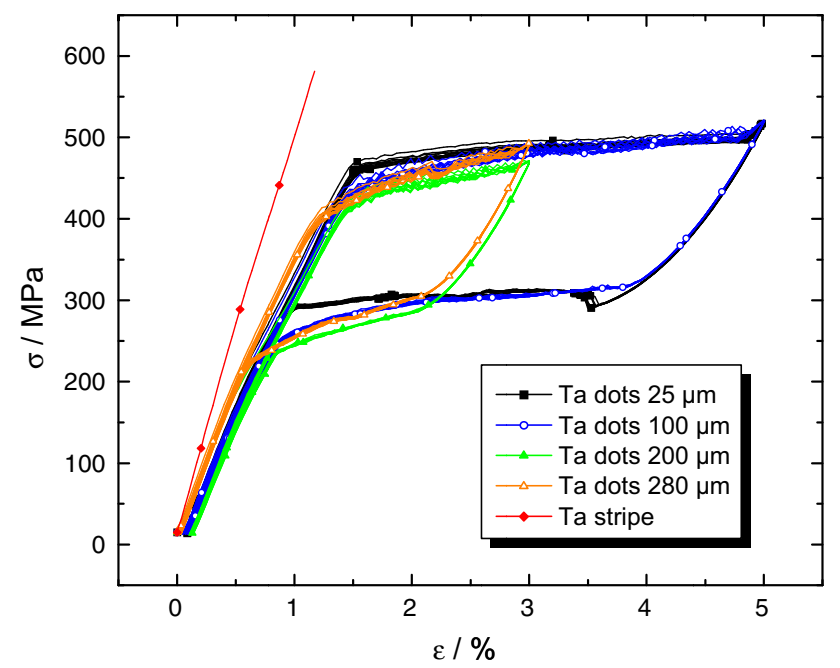

Fig. 4 First ten tensile cycles at $37{ }^{\circ} \mathrm{C}$ of tensile specimen covered with different Ta geometries as shown in Fig. 3. Specimen with Ta dots of 25 and $100 \mu \mathrm{m}$ diameter reveal a flat, smooth forward plateau up to $5 \%$ strain. For specimen with $200-$ and $280-\mu \mathrm{m}$ Ta dots, the transformation is significantly hindered by the Ta layer, indicated by the rugged forward plateau caused by the formation of multiple martensite variants between the Ta dots. Due to the increase in stress concentration, strain was reduced to $3 \%$ to avoid film breakage. For the specimen with a single Ta stripe of $20 \mu \mathrm{m}$ thickness and $250 \mu \mathrm{m}$ width, transformation was completely inhibited

the contour of the dots. This is probably caused by the complex stress fields in the vicinity of the Ta dots and needs to be optimized by FEM analysis.

The cyclic stability of the functional properties of the NiTi structures covered with Ta was also investigated. Figure 5 shows tensile tests up to 250 cycles, (a) for $25-\mu \mathrm{m}$ Ta dots, (b) $280-\mu \mathrm{m}$ Ta dots, (c) a pure NiTi thin film reference, and (d) a commercially available sheet metal, respectively. The specimen with $25-\mu \mathrm{m}$ Ta dots reveals a high stability of the functional properties. Despite a drop in the upper plateau stress from 475 to $430 \mathrm{MPa}$, the slope of the plateau remains rather constant. The lower plateau does not change significantly and the remanent deformation amounts to $0.3 \%$ only after 250 full transformation cycles, indicating a low amount of plastic deformation within the material. In contrast, the functional properties of the commercially available sheet metal change in a more pronounced manner, and a remanent deformation of $0.9 \%$ is observed after 250 cycles. SEM investigation of the laser cut and electropolished sheet metal stripes revealed an inhomogeneity in both thickness and width. A mean value of $50 \mu \mathrm{m}$ thickness and $435 \mu \mathrm{m}$ width were assumed for stress calculation, which results in a slightly lower plateau stress compared to the fabricated sputtered films. However, the comparison of the two materials at hand is difficult due to their differences in microstructure, caused by the different processing routes, such as grain size, size and distribution of precipitates, their different $A_{\mathrm{f}}$ temperatures, and presumably different chemical composition. Processing steps, such as cold or hot rolling, can lead to microstructures with nm-sized grains in bulk materials, which are beneficial for functional stability under fatigue. This effect, however, has not been observed here. DSC measurements of the sputtered NiTi structures covered with Ta revealed a two-step reverse transformation with an intermediate R-phase, and transformation finish temperatures from the martensite phase ranging between 15 and $20{ }^{\circ} \mathrm{C}$. The larger Ta dots limit the NiTi transformation partially in tensile testing, as shown in Fig. 3, but transformation temperatures during stress-free DSC measurements seem to be unaffected compared to the uncovered NiTi thin film. The sputtered NiTi dogbone structures with 280- $\mu \mathrm{m}$ Ta dots were tested up to $3 \%$ strain, and a remanent deformation of $0.1 \%$ after 100 cycles was found. The NiTi reference shows similar behavior compared to the sample with $25-\mu \mathrm{m}$ Ta dots, with a rather good stability of the functional properties and an equal remanent

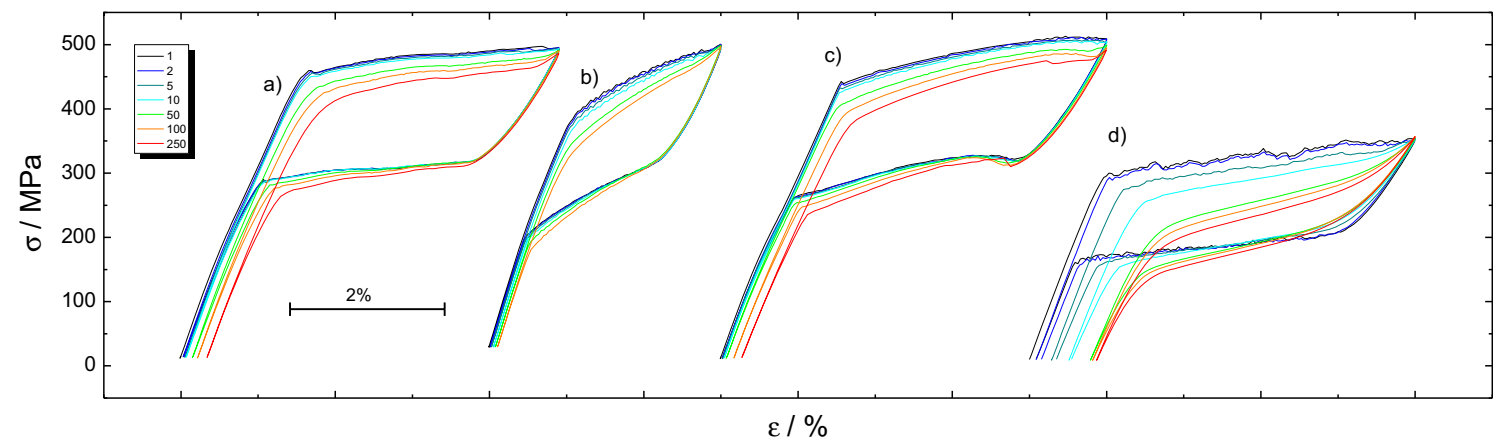

Fig. 5 Cyclic tensile tests at $37^{\circ} \mathrm{C}$ up to 250 cycles. a Ta dots $25 \mu \mathrm{m}$. b Ta dots $280 \mu \mathrm{m}$. c NiTi reference without Ta. d Commercially available sheet metal. a, c Reveal similar functional behavior, also in respect to remanent deformation $(0.33$ and $0.27 \%$, respectively, after 250 cycles to $5 \%$ strain). b Tensile specimen with $280-\mu \mathrm{m}$ Ta dots reveals a higher plateau slope, and a rather rugged plateau, due to the formation of multiple martensite variants between the Ta dots during forward transformation. Remanent deformation was $0.1 \%$ after 100 cycles to $3 \%$. d Commercially available sheet metal reveals lowest cyclic stability and highest remanent strain ( $0.88 \%$ after 250 cycles to $5 \%$ strain) 

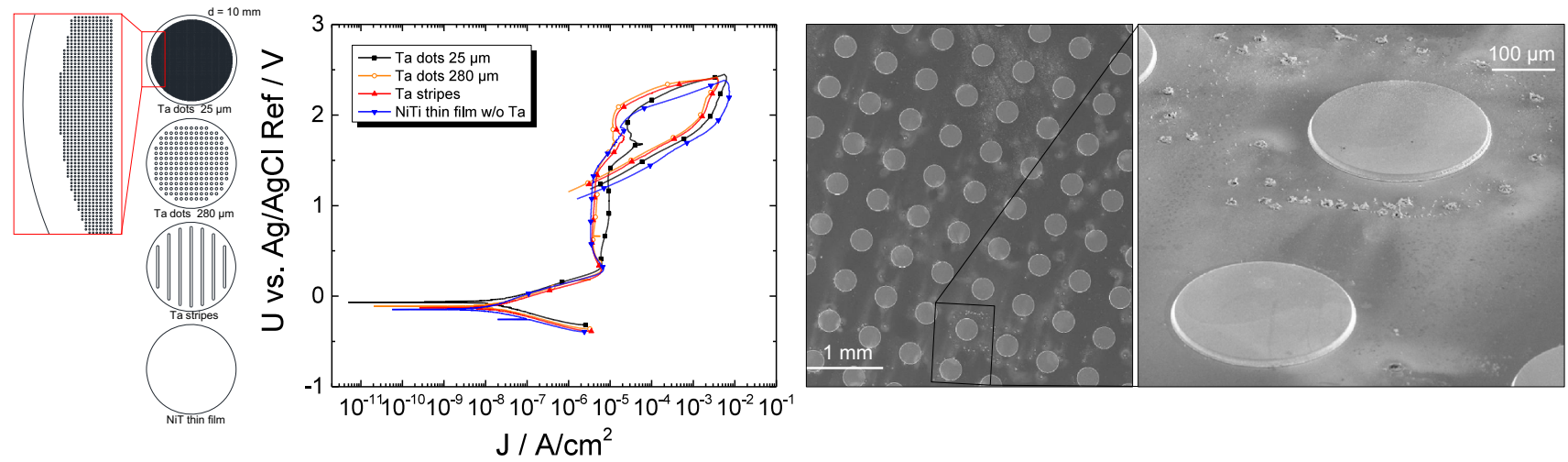

Fig. 6 Corrosion measurement on circular, $1.08 \mathrm{~cm}$ diameter NiTi thin film samples covered with different Ta geometries. All samples reveal high breakdown potentials. SEM images after corrosion tests reveal the deposition of electrolyte constituents, and no signs of pitting corrosion

deformation of $0.3 \%$. The lack of oxide and carbide inclusions in sputtered NiTi thin films further contributes to their good mechanical fatigue performance. It was shown that their fatigue safety limit reaches $1.5 \%$ alternating strain, a significantly improved value compared to standard NiTi materials [37].

The corrosion properties of the NiTi-Ta structures were investigated in Hanks solution according to ASTM standard [32]. Dimensional details of corrosion samples are indicated in Table 1. Corrosion samples with four different Ta geometries were tested: samples covered with $25-\mu \mathrm{m} \mathrm{Ta}$ dots, with $280-\mu \mathrm{m}$ Ta dots, with Ta stripes, and NiTi thin film reference samples without Ta. The amount of dots was chosen so that the Ta surface was kept constant between the samples. Prior to the corrosion tests, the open-circuit potential was measured for $10,000 \mathrm{~s}$. Potentiodynamic scans were performed with a scan rate of $1 \mathrm{mV} / \mathrm{s}$. All samples reveal excellent corrosion resistance independent of the geometry of the Ta layer, with breakdown potentials in the range of $2 \mathrm{~V}$ and rest potentials between 0.08 and $0.14 \mathrm{~V}$, measured versus the $\mathrm{Ag} / \mathrm{AgCl}$ reference electrode (Fig. 6). However, breakdowns do not appear as a discrete jump in corrosion current, but rather a steep increase in slope. The repassivation potential ranged between 1.1 and $1.3 \mathrm{~V}$, passivation currents for the tested $1.08 \mathrm{~cm}$ diameter surface ranged between 3 and $9 \mu \mathrm{A}$. SEM investigations after corrosion tests revealed the deposition of electrolyte constituents, and no signs of pitting corrosion. It is therefore assumed that the increasing currents at voltages higher than $2 \mathrm{~V}$ are not connected to corrosion breakdown, but rather to other processes such as gas formation.

\section{Conclusion and Outlook}

We presented a novel approach to fabricate self-expandable medical components from NiTi with increased radiopacity. The MEMS fabrication process allows for producing fine superelastic NiTi structures, covered locally with arbitrary Ta patterns. NiTi thin films with Ta thicknesses of up to $20 \mu \mathrm{m}$ have been investigated and their X-ray visibility has been shown. The investigation of the mechanical properties of these multilayer structures revealed a strong influence of the Ta geometry on the transformation of the underlying $\mathrm{NiTi}$, with a complete inhibition of transformation in case of a continuous Ta stripe covering $50 \%$ of the NiTi tensile specimen. Samples with patterned Ta dots allowed at least for a partial martensitic transformation between the dots. When the total surface area covered with Ta is further reduced, which is the case for samples with dots of $100 \mu \mathrm{m}$ and smaller, a complete martensitic transformation up to $5 \%$ strain was observed. The array of $25-\mu \mathrm{m}$ Ta dots revealed no significant influence on the superelastic NiTi transformation, the tensile curve being similar to that of pure NiTi thin film and revealing a good cyclic stability of the functional properties with low remanent deformation after 250 full cycles to 5\% strain. However, the Ta dot geometry seems disadvantageous in respect to the inhomogeneity of stress fields generated during the transformation, as well as in respect to the angle between tensile strain direction and the Lüder-like transformation bands. FEM calculations need to be employed to optimize the Ta geometry, and will result in an application-specific compromise between an unrestricted transformation and X-ray visibility. The corrosion resistance of the investigated multilayer structures is excellent and is not affected by the additional Ta layer, with breakdown potentials in the range of $2 \mathrm{~V}$. This exceeds significantly the breakdown acceptance criterion of $0.6 \mathrm{~V}$ [38]. However, the observed behavior does not really imply corrosion breakdown, but rather gas formation [26] and the deposition of electrolyte constituents.

The current study demonstrates promising results of sputtered $\mathrm{NiTi}+$ Ta multilayer devices for medical applications. On the other hand, the study has some limitations: the biocompatibility of the fabricated multilayer structures 
was not addressed, and will have to be investigated in vitro in future work. However, the combination of Ta and NiTi seems to be uncritical, since the multilayers show good corrosion resistance and both $\mathrm{NiTi}$ and $\mathrm{Ta}$ are biocompatible. Also, the multilayer samples showed high passivation potential in the corrosion tests; however, corrosion was not characterized under strain, e.g., under pulsatile testing, and scratch tests were equally not performed.

Acknowledgements The authors acknowledge the contribution of G. Siekmeyer of Admedes Schüssler GmbH (surface finishing of $\mathrm{NiTi}+\mathrm{Ta}$ structures, fruitful discussions) and of Dr. Jens Trentmann from the department of Radiology and Neuroradiology at the UKSH Kiel for radiopacity investigations.

\section{References}

1. Duerig T, Pelton A, Stöckel D (1999) An overview of nitinol medical applications. Mater Sci Eng A 273:149-160

2. Wiesinger B, Stütz A, Schmehl J, Groezinger G, Claussen CD, Wiskirchen J (2012) Comparison of visibility for four self-expanding nitinol bare stents in vitro. Acta Radiol 53:1020-1025

3. Boylan JF (2003) The development of radiopaque nitinol. In: Proceedings SMST. pp 1-6

4. Boylan JF, Cox DL (2004) Radiopaque Nitinol alloys for medical devices, patent US20080027532

5. Diamant V, Koren D, Lotkov A, Sivokha V, Meysner L, Grishkov V, Voronin V (2014) Radiopaque alloy and medical device made of this alloy, patent WO2009085757

6. Lin ZC, Mackiewicz D, Pike K, Boylan JF (2006) Bending fatigue study of radiopaque nitinol. In: Proceedings SMST. pp 79-85

7. Chovan D, Nolan M, Tofali SAM (2015) First principles simulations of elastic properties of radiopaque NiTiPt. J Alloys Compd 630:54-59

8. Li H, Cong Y, Zheng Y, Cui L (2016) In vitro investigation of NiTiW shape memory alloy as potential biomaterial with enhanced radiopacity. Mater Sci Eng C 60:554-559

9. Wang S, Jiang FM, Liu DQ, Cui LS (2014) In situ W-NiTi shape memory alloy composite of high radiopacity. Scr Mater 81:4-7

10. Schaffer JE, Gordon R (2003) Engineering characteristics of drawn filled nitinol tube. In: Proceedings SMST. pp 109-118

11. Wu KH, Ma JL, Pu Z (2000) Constitutional phases and transformation characteristics of Ni41Ti39Ta20 alloy. Mater Sci Forum 327:155-158

12. Ma JL, Wu KH, Pu Z (2000) Microstructure and transformation in behavior of Ni50Ti50-xtax alloys. Mater Sci Forum 327:179-182

13. Nagy P (2015) X-ray analysis of stents and their markers. Period Polytech Mech Eng 59(1):30-34

14. Nagy P (2015) X-ray examination of integrated stent marker. IRBM 36:15-19

15. Steegmüller R, Strobel M, Flaxmeier E, Schüßler A (2004) Micro-welding for improved radiopacity of nitinol stents. In: Proceedings SMST. pp 591-596

16. Stoeckel D, Pelton A, Duerig T (2004) Self expanding nitinol stents-material and design considerations. Eur Radiol 14:292-301

17. Trepanier C, Tabrizian M, Yahia L, Bilodeau L, Piron DL (1998) Effect of modification of oxide layer on NiTi stent corrosion resistance. J Biomed Mater Res 43(4):433-440

18. Wiskirchen J, Venugopalan R, Holton AD, König C, Kramer U, Trübenbach J, Tepe G, Claussen CD, Duda H (2003) Radiopaque markers in endovascular stents-benefit and potential hazards. Fortschr Röntgenstr 175(4):484-488

19. Shabalovskaya SA (2002) Surface, corrosion and biocompatibility aspects of nitinol as an implant material. Biomed Mater Eng 12:69-109

20. Ryhänen J, Niemi E, Serlo W, Niemelä E, Sandvik P, Pernu H, Salo T (1997) Biocompatibility of nickel-titanium shape memory metal and its corrosion behavior in human cell cultures. J Biomed Mater Res 35(4):451-457

21. Shabalovskaya SA, Anderegg J, van Humbeeck J (2008) Critical overview of nitinol surfaces and their modifications for medical applications. Acta Biomater 4(3):447-467

22. Wever DJ, Veldhuizen AG, Sanders MM, Schakenraad JM, van Horn JR (1997) Cytotoxic, allergic and genotoxic activity of a nickel-titanium alloy. Biomaterial 18(16):1115-1120

23. Venugopalan R, Trepanier C (2000) Assessing the corrosion behaviour of nitinol for minimally-invasive device design. Minim Invasive Ther Allied Technol 9(2):67-74

24. Shabalovskaya SA (2004) Comparative corrosion performance of black oxide, sandblasted, and fine-drawn nitinol wires in potentiodynamic and potentiostatic tests: effects of chemical etching and electropolishing. J Biomed Mater Res B 69(2):223-231

25. Shabalovskaya SA, Anderegg J, Laab F, Thiel PA, Rondelli G (2003) Surface conditions of nitinol wires, tubing, and as-cast alloys. J Biomed Mater Res B 65(1):193-203

26. Wohlschlögel M, Lima de Miranda R, Schüßler A, Quandt E (2016) Nitinol—tubing versus sputtered film-microcleanliness and corrosion behavior. J Biomed Mater Res 104(6):1176-1181

27. Wohlschlögel M, Steegmüller R, Schüßler A (2014) Effect of inclusion size and distribution on the corrosion behavior of medical-device grade nitinol tubing. J Mater Eng Perform 23(7):2635-2640

28. Steegmüller R, Wagner C, Fleckenstein T, Schuessler A (2001) Gold coating of nitinol devices for medical applications. Mater Sci Forum 394-395:161-164

29. Steegmüller R, Fleckenstein T, Schüßler A (2006) Surface treatments for obtaining optimized conditions for material combinations with nitinol. Proceedings SMST. pp 227-234

30. Lima de Miranda R, Zamponi C, Quandt E (2013) Micropatterned freestanding superelastic TiNi films. Adv Eng Mater 15(1-2):66-69

31. Bechtold C, Lima de Miranda R, Quandt E (2015) Capability of sputtered micro-patterned NiTi thick films. Shape Mem Superelast 1.3:286-293. doi:10.1007/s40830-015-0029-9

32. ASTM F2129-08 (2001) Standard test method for conducting cyclic potentiodynamic polarization measurements to determine the corrosion susceptibility of small implant devices

33. Jurgeleit T, Quandt E, Zamponi C (2015) Magnetron sputtering a new fabrication method of iron based biodegradable implant materials. Adv Mater Sci Eng. doi:10.1155/2015/294686

34. Matson DW, Merz MD, McClanahan ED (1992) High rate sputter deposition of wear resistant tantalum coatings. J Vac Sci Technol A 10:1791-1796

35. Shaw JA, Kyriakides S (1997) On the nucleation and propagation of phase transformation fronts in a NiTi alloy. Acta Mater 45(2):683-700

36. Sittner P, Liu Y, Novak V (2005) On the origin of Lüders-like deformation of NiTi shape memory alloys. J Mech Phys Solids 53(8):1719-1746

37. Siekmeyer G, Schüßler A, Lima de Miranda R, Quandt E (2014) Comparison of the fatigue performance of commercially produced nitinol samples versus sputter-deposited nitinol. JMEPEG 23:2437-2445

38. Rosenbloom SN (2007) Corrosion testing of nitinol implants per ASTM F 2129-understanding corrosion and interpretation of test results. In: Gilbert J (ed) Medical device materials IV. Palm Desert, pp 223-228 